\title{
Homocysteine, circulating vascular cell adhesion molecule and carotid atherosclerosis in postmenopausal vegetarian women and omnivores ${ }^{\text {is }}$
}

\author{
Ta-Chen Su ${ }^{a, b}$, Jiann-Shing Jeng ${ }^{c}$, Jung-Der Wang ${ }^{\text {a,b }}$, Pao-Ling Torng ${ }^{d}$, \\ Sue-Joan Chang ${ }^{\mathrm{e}}$, Chen-Fang Chen ${ }^{\mathrm{b}}$, Chiau-Suong Liau ${ }^{\mathrm{a}, *}$ \\ a Department of Internal Medicine, National Taiwan University Hospital, College of Medicine, National Taiwan University, \\ No. 7, Chung-Shan South Road, Taipei 100, Taiwan \\ ${ }^{\mathrm{b}}$ Institute of Occupational Medicine and Industrial Hygiene, College of Public Health, National Taiwan University, Taipei, Taiwan \\ ${ }^{\mathrm{c}}$ Department of Neurology, National Taiwan University Hospital, College of Medicine, National Taiwan University, \\ No. 7, Chung-Shan South Road, Taipei 100, Taiwan \\ d Department of Obstetrics and Gynecology, National Taiwan University Hospital, College of Medicine, National Taiwan University, \\ No. 7, Chung-Shan South Road, Taipei 100, Taiwan \\ e Department of Biology, National Cheng Kung University, Tainan, Taiwan
}

Received 13 October 2004; received in revised form 15 April 2005; accepted 22 April 2005

Available online 6 July 2005

\begin{abstract}
Since the adoption of vegetarian diets as a healthy lifestyle has become popular, the cardiovascular effects of long-term vegetarianism need to be explored. The present study aimed to compare the presence and severity of carotid atherosclerosis (CA), and the blood levels of Vitamin B12, homocysteine (Hcy) and soluble vascular cell adhesion molecule-1 (sVCAM-1) between 57 healthy postmenopausal vegetarians and 61 age-matched omnivores. Carotid atherosclerosis, as measured by ultrasound, was found to be of no significant difference between the two groups. Yet, fasting blood glucose, low-density lipoprotein cholesterol, and Vitamin B12 were significantly lower, while Hcy and sVCAM-1 were higher in the vegetarians as comparing with the omnivores. Multivariate regression analysis showed that the level of Vitamin B12 was negatively associated with the level of Hcy. Vegetarianism itself and Hcy level were significantly associated with sVCAM-1 level in univariate analysis; however, after adjustment for covariates, we identified age but not vegetarianism as the determinant of sVCAM-1 level. Multiple linear regression analysis identified age and systolic blood pressure, but not vegetarianism, as determinants of common carotid artery IMT. In conclusion, there was no significant difference in CA between apparently healthy postmenopausal vegetarians and omnivores. The findings of elevated Hcy in vegetarians indicate the importance of prevention of Vitamin B12 deficiency.
\end{abstract}

(C) 2005 Elsevier Ireland Ltd. All rights reserved.

Keywords: Vegetarians; Postmenopausal women; Carotid atherosclerosis; Cell adhesion molecule; Homocysteine

\footnotetext{
is This study has been presented in the 44th Annual Conference on Cardiovascular Disease Epidemiology and Prevention featuring the L.J. Filer Symposium on Prevention of Overweight and Its Consequences Beginning in Youth, in association with the Council on Nutrition, Physical Activity and Metabolism, 3-6 March 2004, San Francisco, CA.

* Corresponding author. Tel.: +886 $223123456 \times 5033$; fax: +886223934176 .

E-mail address: csliau@ @a.mc.ntu.edu.tw (C.-S. Liau).
}

\section{Introduction}

Some studies have shown the harmful effects of adherence to a vegetarian diet. Vegetarian diets contain mainly vegetables, soybean products, and nuts. However, meat- and fish-free diets may be associated with nutritional imbalance. Vitamin B12 deficiency has been associated with elevated total plasma homocysteine (Hcy) level in vegetarians, and is often identified as an issue of concern in this specific group of persons [1-3]. Functional Vitamin B12 deficiency in vegetarians may contribute to hyperhomocysteinemia and 
decreased total antioxidant status, which may partly counteract the beneficial lifestyle of vegetarians [2]. Vitamin B12 and iron status are compromised by a vegetarian diet, and both lower lymphocyte and platelet counts are accompanied by metabolic evidences that indicate Vitamin B12 deficiency [4]. Some studies also did not confirm the protective effects of a vegetarian lifestyle. Vegetarian, especially vegan, diets are relatively low in $\alpha$-linolenic acid and provide little eicosapentaenoic acid (EPA) and docosahexaenoic acid (DHA) which would provide potent cardioprotective effects [5]. As demonstrated in a study on Hong Kong Chinese, the vegetarians have a lower blood or tissue n-3: n-6 polyunsaturated fatty acid ratio in comparing with omnivores [6].

Circulating soluble vascular cell adhesion molecule-1 (sVCAM-1) and intercellular adhesion molecule-1 (sICAM1) have been demonstrated to have a positive association with carotid atherosclerosis (CA) and cardiovascular disease (CVD) [7,8]. However, the above associations have not been tested in vegetarians.

Subclinical extracranial carotid atherosclerosis and carotid intima-media thickness (IMT) measured by carotid ultrasound are strongly associated with CVD [9,10] and its risk factors $[11,12]$. With high reliability and reproducibility $[13,14]$, ultrasound measurements of carotid arteries have been widely used in epidemiological and clinical studies for the evaluation of early CA [9-12]. However, to the best of our knowledge, there is still no report correlating vegetarianism with CA.

Vegetarianism as a healthy lifestyle has become more popular in recent years. It is timely to explore the effects of a vegetarian diet on cardiovascular risks. We chose postmenopausal women for this study because postmenopausal women have higher odds of carotid atherosclerotic plaque compared with premenopausal women [15]. This study was undertaken to determine whether $\mathrm{CA}$, indexed by carotid artery IMT and extracranial carotid artery plaque score, differs between healthy postmenopausal vegetarian women and matched omnivore women. In this study, lipid profiles, fasting glucose, sVCAM-1, sICAM-1, Hcy, and associated certain micronutrients (Vitamins B6, B12 and folate) were also compared in both groups.

\section{Methods}

\subsection{Study subjects}

A total of 57 healthy postmenopausal vegetarians and 61 matched healthy omnivorous postmenopausal women were invited to participate in this study from about 1000 public service volunteers that visited the health center of the Buddhist Compassion Relief Tzu Chi Foundation in Taipei during the years 1999 and 2000. Menopause was defined as a cessation of menstruation cycles for at least 1 year and was verified with a structured questionnaire.
To fulfill the criteria of apparently healthy subjects, women with any of the following conditions were excluded: a history of diabetes mellitus or fasting blood glucose over $126 \mathrm{mg} / \mathrm{dL}$; hyperlipidemia (cholesterol level $\geq 240 \mathrm{mg} / \mathrm{dL}$ or triglyceride level $\geq 200 \mathrm{mg} / \mathrm{dL}$ ); regular alcohol drinking or smoking; any treatment that might affect lipid metabolism; history of CVD, hypertension (systolic blood pressure (SBP) $\geq 160 \mathrm{mmHg}$ or diastolic blood pressure $\geq 95 \mathrm{mmHg}$ ), or use of anti-hypertensive medication; or serum creatinine level $\geq 125 \mu \mathrm{mol} / \mathrm{L}$; thyroid disease; and malignancy of any kind. Vegetarians were defined as exclusive consumption of a vegetarian diet void of meat, fish, and poultry for at least 5 years. Written informed consent was obtained from each participant and this study was approved by the National Taiwan University Hospital.

\subsection{Carotid atherosclerosis assessments}

Maximal carotid IMT and extracranial carotid artery (ECCA) plaque score were used for the assessment of CA. Maximal IMT of the common carotid artery (CCA), bulb, and internal carotid artery (ICA) were measured on both sides using a high-resolution B-mode ultrasonographic system (Hewlett Packard SONO 4500 ultrasound system equipped with a $3-11 \mathrm{MHz}$ real-time B-mode scanner and a $3.6 \mathrm{MHz}$ pulsed-Doppler mode scanner, Andover, MA, USA). The protocol of measurements was described in our previous reports $[12,13]$.

Maximal IMT of the posterior wall of the carotid artery was measured from the leading edge of the first echogenic line to that of the second echogenic line. The examiners (JSJ and TCS) were blinded to the study subjects' health status and risk factors. The inter-observer absolute difference of IMT measurements was $0.13 \pm 0.10$ and $0.11 \pm 0.09 \mathrm{~mm}$ at CCA1 $(0-1 \mathrm{~cm}$ from carotid bifurcation) and CCA2 (1-2 cm from bifurcation), respectively. The intra-observer absolute difference of IMT measurements was $0.14 \pm 0.12$ and $0.12 \pm 0.13 \mathrm{~mm}$ at CCA1 and CCA2, respectively [13].

The scoring method for the extracranial carotid atherosclerotic plaques was described in our previous reports [10,12]. In this study, the sites of examination of the carotid system included the proximal and distal CCA $(>20$ and $<20 \mathrm{~mm}$ proximal to the bulb bifurcation, respectively), bulb, ICA, and external carotid artery bilaterally. The plaque score was computed by summing the plaque grades of all the segments of the carotid arteries studied. Reproducibility of the plaque grade scoring was good with a kappa value of $0.70[10,12]$. All measurements were recorded on super-VHS videotape for subsequent off-line analysis.

\subsection{Blood sample assays}

After fasting for 10-14 h overnight, blood samples were drawn from the antecubital vein. Serum lipids, including total cholesterol, high-density lipoprotein cholesterol (HDL-C), 
and triglyceride, were assayed by enzymatic methods with automatic multi-channel chemical analyzer (Hitachi 7450, Hitachi Corp., Tokyo, Japan) in the central laboratory of the National Taiwan University Hospital. Low-density lipoprotein cholesterol (LDL-C) was calculated by Friedewald's formula.

Blood samples were first centrifuged at $3000 \mathrm{rpm}$ for 15 min within $30 \mathrm{~min}$ of collection, and then stored at $-70^{\circ} \mathrm{C}$ until assayed. Plasma levels of Vitamin B12 and folate were measured by microparticle enzyme immonoassay technology with $\mathrm{AxSYM}^{\circledR}$ B12 and Folate kits (Abbott Laboratories, Chicago, IL). Plasma Vitamin B6 concentration was determined by HPLC with fluorometric detection (Hitachi, Tokyo, Japan) [16] while plasma total homocysteine was determined by competitive immunoassay with IMMULITE ${ }^{\circledR}$ Homocysteine kit (Diagnostic Products Corporation, Los Angeles, CA). Serum levels of sVCAM-1 and sICAM1 were measured by enzyme linked immunosorbent assay with commercial kits (R\&D Systems Inc., Minneapolis, $\mathrm{MN})$.

\subsection{Statistical analysis}

In the data analysis, demographic and clinical characteristics and the clinical measurements were compared between vegetarians and omnivores. Continuous variables were expressed as mean \pm standard deviation. Both $t$-test and ANOVA test were used to make comparisons. For categorical data, $\chi^{2}$ - test was used. Since the distributions of Vitamin B12, B6, and sVCAM-1 were not normal, Wilcoxon-Mann-Whitney test was used for analysis.

The total ECCA plaque score and the averaged values of bilateral IMTs at the CCA, bulb, and ICA were compared between vegetarians and omnivores. Because CVD risk factors influence the carotid IMT significantly, the comparisons of average IMTs were performed with values of least-square mean \pm standard error after adjustment for age, LDL-C, SBP, and BMI. Plaque scores were also stratified into three levels $(0,1-3,>3)$ to evaluate the differences between vegetarians and omnivores.
Multiple linear regression analysis with stepwise procedure was conducted to evaluate the predictive value of Vitamin B12 on the levels of Hcy and sVCAM-1, respectively. The predictive value of Hcy on the level of SVCAM-1 also was calculated. The significant determinants of IMT of CCA were also evaluated by using multiple linear regression models to estimate the effects of vegetarianism after controlling major CVD risk factors (age, SBP, LDL-C, fasting glucose, BMI and menopausal year). Hcy was also taken into account with the vegetarian status in the multivariate analysis models. All statistical analyses were performed with SAS statistical software (Version 8.2, SAS Institute, Cary NC, USA).

\section{Results}

Baseline characteristics of the vegetarian group and the controlled omnivore group are shown in Table 1. In comparison with the omnivore group, the levels of fasting serum glucose, HDL-C and LDL-C were significantly lower in the vegetarian group. The duration of vegetarian diet averaged $10.4 \pm 4.2$ years for the vegetarian group. Most vegetarians adopted a vegan type diet (taking no egg or milk). The ratio of lactovegetarians/vegans was $6 / 51$ in this study. The age of menopause was 2.1 years younger and the year after menopause was 3.8 years longer for the vegetarian group. The BMI was not statistically different between two groups.

As shown in Table 2, the concentration of Vitamin B12 was lower and the level of Hcy was higher in the vegetarian group. sVCAM-1 was higher, but sICAM-1 was not higher in vegetarians than in omnivores. However, levels of folate, Vitamin B6, and serum creatinine were not different between the two groups. In Table 3, carotid IMT (measured at bulb, CCA and ICA, and the average) and the ECCA plaque scores were not significantly different between the two groups of study subjects. The adjusted average of IMT was also not significantly different between the two groups.

In Table 4, multivariate linear regression analysis showed that vegetarians were positively and levels of Vitamin B12

Table 1

Demographic and clinical characteristics of the vegetarian and omnivore groups

\begin{tabular}{lccr}
\hline Characteristics & Vegetarian group $(n=57)$ & Omnivore group $(n=61)$ & $P$ \\
\hline Average age (years) & $59.2 \pm 6.4$ & $57.7 \pm 5.1$ & 0.178 \\
Duration of vegetarian (years) & $10.4 \pm 4.2$ & - & - \\
Age of menopause (years) & $48.0 \pm 5.0$ & $50.1 \pm 4.2$ & 0.017 \\
Years after menopause (years) & $11.4 \pm 6.1$ & $7.6 \pm 6.2$ & 0.002 \\
Body mass index (kg/m ${ }^{2}$ ) & $23.0 \pm 2.7$ & $23.5 \pm 2.7$ & 0.300 \\
Systolic blood pressure (mmHg) & $127.8 \pm 15.7$ & $126.7 \pm 14.8$ & 0.699 \\
Diastolic blood pressure (mmHg) & $71.1 \pm 7.2$ & $73.0 \pm 9.4$ & 0.220 \\
Pulse pressure (mmHg) & $56.6 \pm 12.4$ & $53.6 \pm 11.6$ & 0.179 \\
Cholesterol (mmol/L) & $4.9 \pm 0.8$ & $5.5 \pm 0.7$ & $<0.001$ \\
Triglyceride (mmol/L) & $1.4 \pm 0.7$ & $1.3 \pm 0.6$ & 0.267 \\
HDL-C (mmol/L) & $1.5 \pm 0.3$ & $1.7 \pm 0.4$ & 0.002 \\
LDL-C (mmol/L) & $2.8 \pm 0.7$ & $3.2 \pm 0.7$ & 0.002 \\
Fasting blood glucose (mmol/L) & $5.0 \pm 0.5$ & $5.2 \pm 0.5$ & 0.009 \\
\hline
\end{tabular}


Table 2

Plasma concentrations of homocysteine, Vitamins B6 and B12, folate and cell adhesion molecules in vegetarians and omnivores

\begin{tabular}{|c|c|c|c|}
\hline & Vegetarian group & Omnivore group & $P$ \\
\hline Folate $(\mathrm{nmol} / \mathrm{L})$ & $22.0 \pm 7.5$ & $22.7 \pm 6.1$ & 0.595 \\
\hline Vitamin B12 (pmol/L) ${ }^{\mathrm{a}}$ & $265.2 \pm 179.3$ & $380.3 \pm 199.4$ & $<0.001$ \\
\hline Vitamin B6 $(\mathrm{nmol} / \mathrm{L})^{\mathrm{a}}$ & $121.8 \pm 63.1$ & $108.8 \pm 48.2$ & 0.890 \\
\hline Homocysteine $(\mu \mathrm{mol} / \mathrm{L})$ & $11.0 \pm 3.3$ & $9.0 \pm 2.1$ & $<0.001$ \\
\hline sVCAM-1 (ng/mL) ${ }^{\mathrm{a}}$ & $724.7 \pm 418.3$ & $547.5 \pm 259.9$ & 0.033 \\
\hline sICAM-1 (ng/mL) & $277.6 \pm 111.3$ & $252.2 \pm 70.2$ & 0.145 \\
\hline Creatinine $(\mu \mathrm{mol} / \mathrm{L})$ & $71.5 \pm 8.0$ & $72.5 \pm 7.5$ & 0.373 \\
\hline
\end{tabular}

${ }^{\text {a }}$ Analysis with Wilcoxon-Mann-Whitney test.

were negatively associated with levels of Hcy, but not sVCAM-1. Hcy had a borderline significant association with sVCAM-1 levels; however, we identified age but not vegetarianism as the determinant of SVCAM-1 levels after adjustment for associated covariates.

In Table 5, after adjustment for the covariates, multiple linear regression analysis identified age, fasting glucose, and SBP as the determinants of CCA IMT. Vegetarianism, sVCAM-1 and Hcy were not significant determinants of CCA IMT. However, we found there was a trend of significance for vegetarians on CCA IMT in Model 3. In Table 6, the multivariate logistic regression models found that age, years after menopause, and SBP were the significant risk factors for CA (higher ECCA score $\geq$ upper tertile). However, only age was identified as significant risk for higher IMT (IMT $\geq$ upper tertile).

\section{Discussion}

In the present study we showed that CA was not significantly different between vegetarians and omnivores in apparently healthy postmenopausal Chinese women. However, we found that plasma Hcy and sVCAM-1 were elevated and plasma Vitamin B12 was lower in vegetarians as compared with omnivores, which seemed to be contradictory to

Table 3

Carotid artery intima-media thickness (IMT) and extracranial carotid artery (ECCA) plaque score of the vegetarian and the omnivore groups

\begin{tabular}{|c|c|c|c|}
\hline & Vegetarian group & Omnivore group & $P$ \\
\hline \multicolumn{4}{|l|}{ IMT } \\
\hline CCA (mm) & $0.773 \pm 0.187$ & $0.739 \pm 0.132$ & 0.421 \\
\hline Bulb (mm) & $0.941 \pm 0.279$ & $0.938 \pm 0.308$ & 0.902 \\
\hline ICA (mm) & $0.651 \pm 0.139$ & $0.669 \pm 0.121$ & 0.171 \\
\hline IMT, average & $0.789 \pm 0.173$ & $0.772 \pm 0.159$ & 0.910 \\
\hline IMT, average after adjusted ${ }^{\text {a }}$ & $0.790 \pm 0.021$ & $0.771 \pm 0.020$ & 0.526 \\
\hline ECCA total score ${ }^{b}$ & $0.772 \pm 1.476$ & $0.754 \pm 1.337$ & 0.583 \\
\hline \multicolumn{4}{|l|}{ ECCA score } \\
\hline 0 & $36(63.2 \%)$ & $38(62.3 \%)$ & 0.866 \\
\hline $1-3$ & $16(28.1 \%)$ & $19(31.2 \%)$ & \\
\hline$>3$ & $5(8.8 \%)$ & $4(6.6 \%)$ & \\
\hline
\end{tabular}

a Values are least-square mean \pm standard error after adjustment for age, BMI, LDL-C, and fasting blood glucose.

b Analysis with Wilcoxon-Mann-Whitney test.

Table 4

Multiple linear regression analyses for individual risk factors on the level of homocysteine $(\mu \mathrm{mol} / \mathrm{L})$ and sVCAM-1 $\left(10^{-2} \mathrm{ng} / \mathrm{mL}\right)$

\begin{tabular}{|c|c|c|c|c|}
\hline \multirow[t]{2}{*}{ Variable } & \multicolumn{2}{|l|}{ Homocysteine } & \multicolumn{2}{|l|}{ sVCAM } \\
\hline & $\beta$ (S.E.) & $P$ & $\beta$ (S.E.) & $P$ \\
\hline \multicolumn{5}{|l|}{ Model 1} \\
\hline Vegetarians & $1.51(0.55)$ & 0.0068 & $1.06(0.67)$ & 0.1181 \\
\hline Age (years) & $0.01(0.05)$ & 0.7686 & $0.12(0.06)$ & 0.0575 \\
\hline \multicolumn{5}{|l|}{ Model 2} \\
\hline Vitamin B12 $\left(\times 10^{-2} \mathrm{pmol} / \mathrm{L}\right)$ & $-0.49(0.11)$ & $<0.0001$ & $-0.19(0.13)$ & 0.1661 \\
\hline Age (years) & $0.05(0.05)$ & 0.3120 & $0.16(0.06)$ & 0.0108 \\
\hline \multicolumn{5}{|l|}{ Model 3} \\
\hline Homocysteine $(\mu \mathrm{mol} / \mathrm{L})$ & & & $0.21(0.12)$ & 0.0719 \\
\hline Age (years) & & & $0.13(0.06)$ & 0.0408 \\
\hline
\end{tabular}

Models were expressed after adjustment for age, body mass index, low-density lipoprotein cholesterol, fasting sugar, and systolic blood pressure, in addition to above variables. Model 1 includes vegetarians and age, model 2 includes Vitamin B12 and age, and model 3 includes homocysteine and age. 
Table 5

Multiple linear regression analysis for the common carotid artery intima-media thickness (IMT)

\begin{tabular}{|c|c|c|c|c|c|c|}
\hline \multirow[t]{3}{*}{ Variable } & \multicolumn{6}{|c|}{$\operatorname{IMT}\left(\times 10^{-2} \mathrm{~mm}\right)$} \\
\hline & \multicolumn{2}{|l|}{ Model 1} & \multicolumn{2}{|l|}{ Model 2} & \multicolumn{2}{|l|}{ Model 3} \\
\hline & $\beta \pm$ S.E. & $P$ & $\beta \pm$ S.E. & $P$ & $\beta \pm$ S.E. & $P$ \\
\hline Vegetarians & $4.48 \pm 2.83$ & 0.116 & $4.80 \pm 3.01$ & 0.114 & $4.97 \pm 2.86$ & 0.085 \\
\hline Homocysteine $((\mathrm{mol} / \mathrm{L})$ & - & - & $0.38 \pm 0.52$ & 0.465 & - & - \\
\hline VCAM-1 $\left(\times 10^{-2} \mathrm{ng} / \mathrm{mL}\right)$ & - & - & - & - & $0.5 \pm 0.4$ & 0.252 \\
\hline Age (years) & $0.83 \pm 0.25$ & 0.001 & $0.92 \pm 0.26$ & 0.001 & $0.88 \pm 0.26$ & 0.001 \\
\hline Systolic BP (mmHg) & $0.22 \pm 0.10$ & 0.025 & $0.22 \pm 0.10$ & 0.029 & $0.22 \pm 0.10$ & 0.022 \\
\hline Fasting glucose $(\mathrm{mmol} / \mathrm{L})$ & $7.39 \pm 2.88$ & 0.013 & $7.39 \pm 3.06$ & 0.018 & $7.03 \pm 2.88$ & 0.021 \\
\hline Adjusted $R^{2}$ & 0.239 & & 0.247 & 0.241 & 0.241 & \\
\hline
\end{tabular}

The regressions coefficient ( $\beta \pm$ S.E.) and $P$ value are indicated. Model 1 excludes homocysteine and VCAM-1 in the multivariate analysis. Model 2 excludes VCAM-1 and model 3 excludes homocysteine, respectively. All models are after adjustment for all above covariates, body mass index, years after menopause, and LDL-C levels. VCAM-1, vascular cell adhesion molecule-1.

the common belief that a vegetarian diet is beneficial to CVD. Thus, all the evidence and inference should be under careful scrutiny.

\subsection{Vegetarians and carotid atherosclerosis}

We could not demonstrate any significant beneficial effects of vegetarian diets on CA in this study. A consistent effect of increased SBP, fasting glucose, and age on IMT was found after multiple linear regression analysis, which corroborated the current hypothesis of atherosclerosis and validated this study. Our previous studies and other reports demonstrated that SBP, hypertension, fasting glucose, and age are important predictors for carotid IMT and CA [10-12,15,17]. The longer duration of years after menopause in vegetarians was associated with earlier atherosclerosis and might partially attenuate our major findings in this study.

\subsection{Vegetarians and lipids}

This study found advantageous cardiovascular effects of a vegetarian diet on plasma cholesterol and LDL-C, which are in line with previous reports [18-20]. Vegetarian diets contain less total fat, especially saturated fat, and less cholesterol (none in vegan diets), which may contribute to lower levels of cholesterol and LDL-C compared with controlled omnivores $[18,19]$. In vegetarians, in addition to elevation in Hcy and SVCAM-1, the findings of lower levels of HDL-C in this study also could counterbalance the beneficial effects of lower LDL-C from vegetarian diet. A study of Chinese people living in Hong Kong revealed similar results [6].

\subsection{Vitamin B12, homocysteine, and SVCAM-1 in vegetarians}

In vegetarians, Hcy was reported to be elevated [1-4]. This finding was confirmed in our study and could be attributed to the relative lower levels of Vitamin B12, which was corroborated by the simultaneous measurements of Vitamin B6 and folate. In our study, multivariate regression analysis also demonstrated a strong inverse association between Hcy and Vitamin B12 levels. Plasma Hcy has emerged as a risk factor for CVD [21], and has been associated with ECCA IMT in the Atherosclerosis Risk in Community Study [22]. Though we could not find significant effects of plasma levels of Hcy on carotid IMT in this study, the elevation of Hcy in vegetarians may exert certain adverse effects on CA.

This study demonstrates a significant association between levels of Hcy and sVCAM-1, and between vegetarians and sVCAM-1; however, these relationships were attenuated after adjustment for associated covariates. Age, not being vegetarian, was found to be the major determinant of SVCAM-1, which echoed the findings of Richter et al. that sVCAM1 is an age-dependent parameter independent of vegetarian

Table 6

Multivariate logistic regression analysis for carotid atherosclerosis

\begin{tabular}{lll}
\hline & IMT $\geq$ upper tertile, OR $(95 \% \mathrm{CI})$ & ECCA score $\geq$ upper tertile, OR $(95 \%$ CI $)$ \\
\hline Vegetarians & $0.844(0.408,1.744)$ & $0.964(0.457,2.034)$ \\
Age (years) & $1.090(1.019,1.166)^{*}$ & $1.192(1.101,1.290)^{\ddagger}$ \\
Years after menopause & $1.048(0.988,1.112)$ & $1.101(1.033,1.172)^{\ddagger}$ \\
Body mass index $\left(\mathrm{kg} / \mathrm{m}^{2}\right)$ & $1.089(0.948,1.252)$ & $1.058(0.919,1.217)$ \\
Systolic BP $(\mathrm{mmHg})$ & $1.019(0.995,1.045)$ & $1.026(1.000,1.053)^{*}$ \\
\hline
\end{tabular}

Models are after adjusting all above covariates, LDL-C, and fasting glucose. IMT, mean value of intima-media thickness; ECCA score, total extracranial carotid atherosclerosis score.

${ }^{*} P<0.05$.

$\ddagger P<0.005$. 
status and cardiovascular risk factors [23]. Hcy has been proved to activate the transcription factor NF-кB in vascular smooth muscle cells and thereby increase the expression of sVCAM-1 [24]. Hcy may contribute to the pathogenesis of atherosclerosis through increased monocyte and T-cell adhesion to human aortic endothelial cells [25]. Thus, the elevated Hcy and VCAM-1 in vegetarians in this study indicate that vegetarianism is not always healthy in nature; at least the issue of Vitamin B12 deficiency deserves further attention.

\subsection{Study limitations}

There are some limitations for this study. The crosssectional design may limit the causal inference. The participants were recruited from healthy volunteers, but not a randomized population may under-estimate the true effects of a long-term vegetarian diet. Moreover, our subjects were in general only about 8 years after menopause, which might not be long enough to develop a significant atherosclerosis in a regular Chinese diet. In other words, we might not have sufficient induction time to observe such a difference. Thus, over-matching might partially explain the negative findings of no difference in CA and carotid IMT. The small sample size of this study may not have had sufficient statistical power to detect a difference between vegetarians and omnivores.

\section{Conclusions}

There was no significant difference in CA between apparently healthy postmenopausal vegetarians and omnivores. In apparently healthy postmenopausal vegetarians, elevation in Hcy and sVCAM-1 levels may attenuate the potential beneficial effects of a vegetarian diet on plasma lipids which may possibly lower the development of CA. In adopting a vegetarian diet as a mode of healthy lifestyle, one should pay special attention to the prevention of Vitamin B12 deficiency, as suggested by Haddad et al. [26].

\section{Acknowledgements}

This study was supported by a grant from the National Taiwan University Hospital, Taipei, Taiwan (NTUH 91-S049) and partly support by National Science Council, Taiwan (NSC 90-2320-B-002-178).

\section{References}

[1] Hung CJ, Huang PJ, Lu SC, Li YH, Huang HB, Lin BF, et al. Plasma homocysteine levels in Taiwanese vegetarians are higher than those of omnivores. J Nutr 2002;132:152-8.

[2] Herrmann W, Schorr H, Purschwitz K, Rassoul F, Richter V. Total homocysteine, vitamin B12, and total antioxidant status in vegetarians. Clin Chem 2001;47:1094-101.
[3] Mezzano D, Muñoz X, Martínez C, Cuevas A, Panes O, Aranda E, et al. Vegetarians and cardiovascular risk factors: hemostasis, inflammatory markers and plasma homocysteine. Thromb Haemost 1999;81:913-7.

[4] Obeid R, Geisel J, Schorr H, Hubner U, Herrmann W. The impact of vegetarianism on some haematological parameters. Eur J Haematol 2002;69:275-9.

[5] Davis BC, Kris-Etherton PM. Achieving optimal essential fatty acid status in vegetarians: current knowledge and practical implications. Am J Clin Nutr 2003;78(Suppl):640S-6S.

[6] Lee HY, Woo J, Chen ZY, Leung SF, Peng XH. Serum fatty acid, lipid profile and dietary intake of Hong Kong Chinese omnivores and vegetarians. Eur J Clin Nutr 2000;54:76873.

[7] Rohde LE, Lee RT, Rivero J, Jamacochian M, Arroyo LH, Briggs $\mathrm{W}$, et al. Circulating cell adhesion molecules are correlated with ultrasound-based assessment of carotid atherosclerosis. Arterioscler Thromb Vasc Biol 1998;18:1765-70.

[8] Price DT, Loscalzo J. Cellular adhesion molecules and atherogenesis. Am J Med 1999;107:85-97.

[9] O’Leary DH, Polak JF, Kronmal RA, Manolid TA, Burke GL, Wolfson Jr SK, et al. Carotid-artery intima and media thickness as a risk factor for myocardial infarction and stroke in older adults. Cardiovascular Health Study Collaborative Research Group. N Engl J Med 1999;340:14-22.

[10] Jeng JS, Chung MY, Yip PK, Hwang BS, Chang YC. Extracranial carotid atherosclerosis and vascular risk factors in different types of ischemic stroke in Taiwan. Stroke 1994;25:198993

[11] Heiss G, Sharrett AR, Barnes R, Chambless LE, Szklo M, Alzola C. Carotid atherosclerosis measured by B-mode ultrasound in populations: associations with cardiovascular risk factors in the ARIC study. Am J Epidemiol 1991;134:250-6.

[12] Su TC, Jeng JS, Chien KL, Sung FC, Hsu HC, Lee YT. Hypertension status is the major determinant of carotid atherosclerosis: a community-based study in Taiwan. Stroke 2001;33:226571.

[13] Su TC, Jeng JS, Chien KL, Torng PL, Sung FC, Lee YT. Measurement reliability of common carotid artery intima-media thickness by ultrasonographic assessment. J Med Ultrasound 1999;7:739.

[14] Persson J, Wikstrand J, Israelsson B, Formgren J, Berglund G. Noninvasive quantification of atherosclerotic lesions. Reproducibility of ultrasonographic measurement of arterial wall thickness and plaque size. Arterioscler Thromb 1992;12:261-6.

[15] Sutton-Tyrrell K, Lassila HC, Meilahn E, Bunker C, Matthews KA, Kuller LH. Carotid atherosclerosis in premenopausal and postmenopausal women and its association with risk factors measured after menopause. Stroke 1998;29:1116-21.

[16] Kimura M, Kanehira K, Yokoi K. Highly sensitive and simple liquid chromatographic determination in plasma of B-6 vitamers, especially pyridoxal-5'-phosphate. J Chromatogr A 1996;722:269301.

[17] Folsom AR, Eckfeldt JH, Weitzman S, Ma J, Chambless LE, Barnes RW, et al. Relation of carotid artery wall thickness to diabetes mellitus, fasting glucose and insulin, body size, and physical activity. Atherosclerosis risk in communities (ARIC) Study Investigators. Stroke 1994;25:66-73.

[18] Pan WH, Chin CJ, Sheu CT, Lee MH. Hemostatic factors and blood lipids in young Buddhist vegetarians and omnivores. Am J Clin Nutr 1993;58:354-9.

[19] Sacks FM, Castelli WP, Donner A, Kass EH. Plasma lipids and lipoproteins in vegetarians and controls. N Engl J Med 1975;292:1148-51.

[20] Lu SC, Wu WH, Lee CA, Chou HF, Lee HR, Huang PC. LDL of Taiwanese vegetarians are less oxidizable than those of omnivores. J Nutr 2000;130:1591-6. 
[21] Clarke R, Daly L, Robinson K, Naughten E, Cahalane S, Fowler $\mathrm{B}$, et al. Hyperhomocysteine: an independent risk factor for vascular disease. N Engl J Med 1991;324:1149-55.

[22] Mailinow MR, Nieto FJ, Szklo M, Chambless LE, Bond G. Carotid artery intimal-medial wall thickening and plasma homocyst(e)ine in asymptomatic adults. The Atherosclerosis Risk in Communities Study. Circulation 1993;87:1107-13.

[23] Richter V, Rassoul F, Purschwitz K, Hentschel B, Reuter W, Kuntze T. Circulating vascular cell adhesion molecules VCAM1, ICAM-1, and E-selectin in dependence on aging. Gerontology 2003;49:293-300
[24] Welch GN, Upchurch Jr GR, Farivar RS, Pigazzi A, Vu K, Brecher P. Homocysteine-induced nitric oxide production in vascular smooth muscle cells by NF-(kappa) B-dependent transcriptional activation of Nos2. Proc Assoc Am Physicians 1998;110:2231.

[25] Koga T, Claycombe K, Meydani M. Homocysteine increases monocyte and T-cell adhesion to human aortic endothelial cells. Atherosclerosis 2002;161:365-74.

[26] Haddad EH, Sabaté J, Whitten CG. Vegetarian food guide pyramid: a conceptual framework. Am J Clin Nutr 1999;70(Suppl):615S9S. 